\title{
An alternative chest tube placement after uniportal video-assisted thoracic surgery
}

\author{
Alessandro Palleschi ${ }^{1}$, Paolo Mendogni ${ }^{1}$, Alessio Vincenzo Mariolo ${ }^{1}$, Mario Nosotti $^{1,2}$, Lorenzo Rosso ${ }^{1}$ \\ ${ }^{1}$ Thoracic Surgery and Lung Transplant Unit, Fondazione IRCCS Ca' Granda Ospedale Maggiore Policlinico, Milan, Italy; ${ }^{2}$ Department of \\ Pathophysiology and Transplantation, Università degli Studi di Milano, Milan, Italy \\ Correspondence to: Paolo Mendogni, MD, PhD. Thoracic Surgery and Lung Transplant Unit, Fondazione IRCCS Ca' Granda Ospedale Maggiore \\ Policlinico, University of Milan, Via Francesco Sforza, 35, Milan, 20122, Italy. Email: paolo.mendogni@unimi.it.
}

Submitted Jul 26, 2017. Accepted for publication Oct 23, 2017.

doi: $10.21037 /$ jtd.2018.04.108

View this article at: http://dx.doi.org/10.21037/jtd.2018.04.108

\section{Introduction}

The field of video-assisted thoracic surgery (VATS) is still in continuous development. The advent of uniportal technique has added a versatile tool for thoracic surgeons; currently, it is used for minor thoracic procedures including lung wedge resections up to complex thoracic operations (i.e., chest wall resection, pneumonectomy or bronchoplastic resection) demonstrating the versatile nature of this technique (1).

The main potential advantage claimed by surgeons who support uniportal VATS is the lower post-operative pain if compared with multiport VATS as well as reduction of morbidity, faster recovery and shorter hospital stay. Nevertheless, a widespread adoption of uniportal VATS is hampered by some limitations due to a single thoracoscopic access, such as a lack of exposure and crowding of instrumentations making surgical manoeuvring more technically demanding (2).

We tried and tested the uniportal approach and found it very convincing. We believe that the benefits to the patient are far in excess the challenges for the surgeon; however, these challenges become more affordable after an appropriate learning curve. In our opinion, what we consider a problem in se is the correct placement of the pleural drainage at the end of the procedure. The chest tube should be inserted to reach the thoracic apex in order to drain air. At the same time, it should prevent the collection of effusions in the pleural cavity (3). The uniportal technique contemplates placing the tube through the thoracotomy, using the same via, from skin to pleura (4). We are not confident with this approach for several reasons: firstly, the suboptimal angulation between the chest wall and the pleural cavity could create tube kinking, wrong positioning, loss of suction and residual idro-pneumothorax. Secondly, the passage of the chest tube directly through the mini-invasive access could impair the correct reconstruction of muscular plane and may compromise the subcutaneous and skin suturing with postoperative unaesthetic results. Finally, a patent passage between pleural cavity and subcutaneous tissue is a potential source of air/fluid collection and infection.

We propose a feasible, safety and easy-performing technique for chest tube placement after uniportal VATS procedures that may prevent all potential drawbacks described above.

\section{Technical notes}

At the end of the surgical procedure, a $1 \mathrm{~cm}$ skin incision is practiced about $3-5 \mathrm{~cm}$ caudal to the thoracotomy, approximately on the projection of two intercostal spaces below. A curved Klemmer forceps is used to practice blunt dissection under the muscular plane, creating a tunnel along the chest wall (Figure 1A). Then the proximal end of the chest tube is firmly held and easily passed through the newdedicated tunnel (Figure 1B).

Measuring the length from the skin incision to the apex of the pleural cavity it is possible to estimate how far the chest tube should be inserted under direct vision through the intercostal incision used for uniportal procedure (Figure 2A). The tunnel represents a stable fulcrum that makes the correct positioning of the chest tube easier. Then the tube can be anchored with stitches through the 

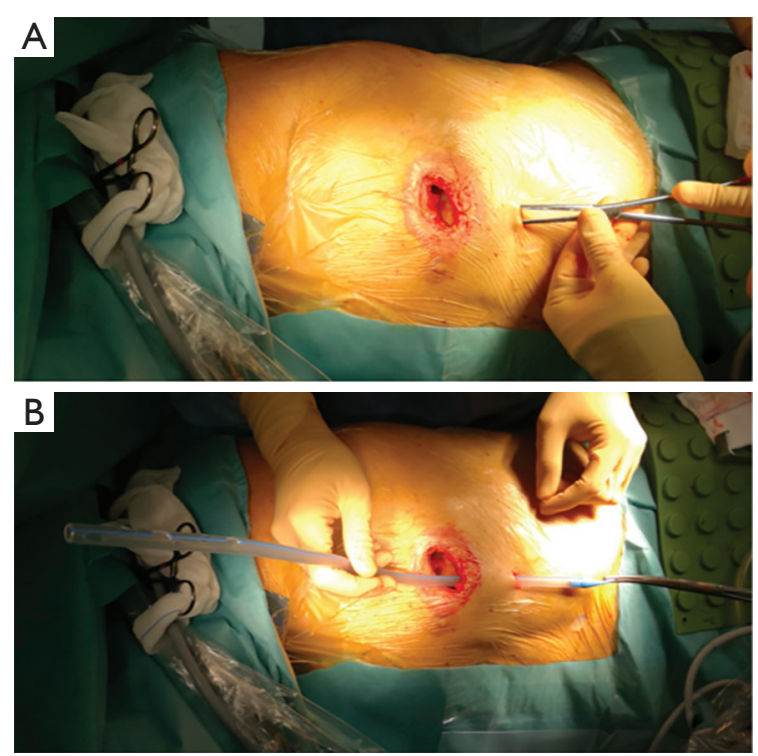

Figure 1 Tunnel technique for placing chest tube. (A) Introduction of the curved Klemmer trough the new skin incision. Blunt dissection is then performed under muscular plane to create the tunnel along the chest wall to reach the thoracotomy access; (B) chest tube is held distally and easily passed through the new-dedicated tunnel. skin as usual. The thoracotomy wound can be carefully closed in layers without interruptions in the deep planes (Figure $2 B$ ) and the skin reconstructed with an intradermic suture (Figure 2C). Finally, the tube drainage can be attached to a digital drainage device, setting a $-20 \mathrm{~cm} \mathrm{H}_{2} \mathrm{O}$ suction to avoid the fluid basal collection.

\section{Conclusions}

Chest tube placement following thoracic surgery is strictly recommended. It facilitates lung re-expansion, allows postoperative pleural effusion evacuation and, in case of any air leaks, it prevents the development of pneumothorax. Even if several controversies exist about the management of the chest tube in the postoperative period, it has been proved that the drainage misplacement can induce infections, pulmonary atelectasis and incomplete lung reexpansion impairing post-operative recovery and implicating longer hospital stay (5). Uniportal VATS approach makes the correct chest tube placement potentially challenging. The sketches of the Figure 3 schematize the trajectory that
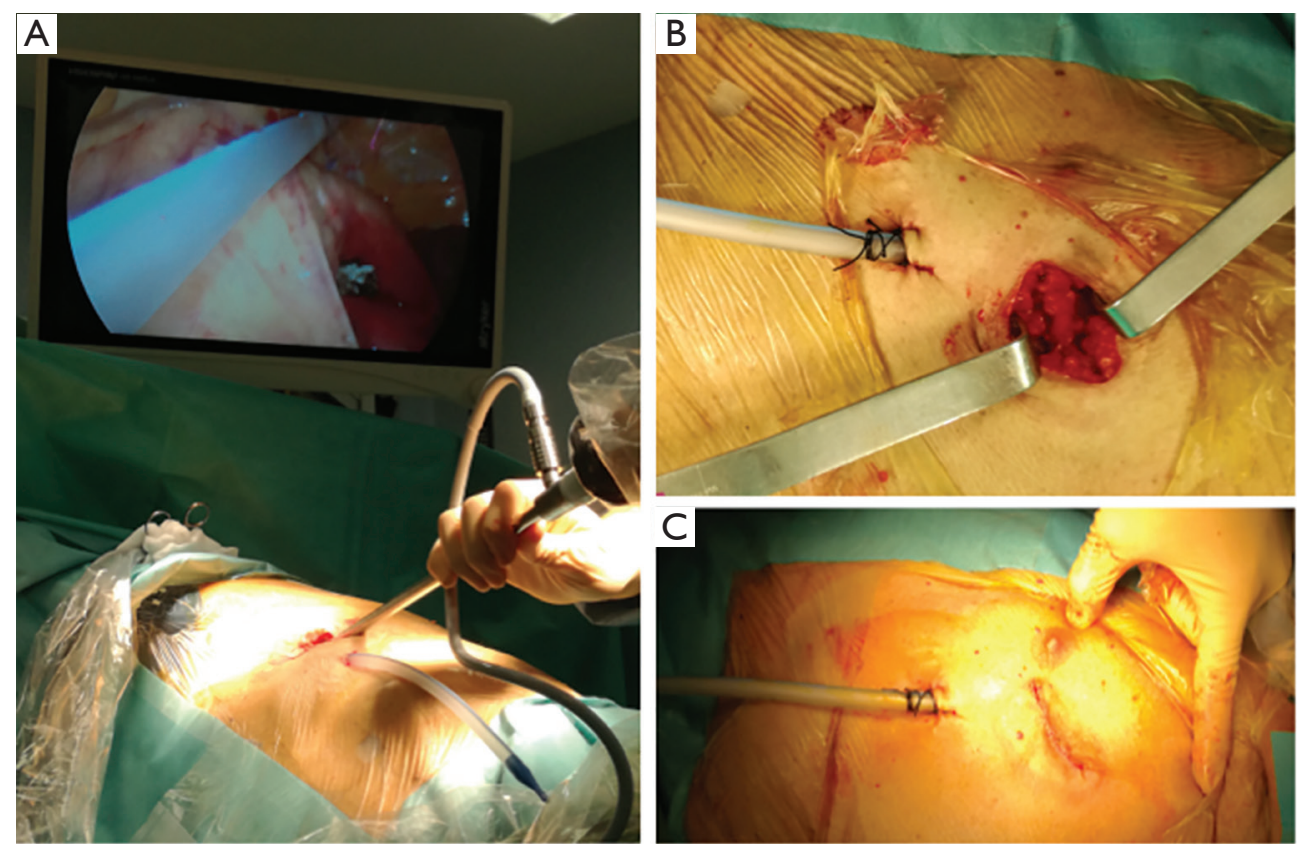

Figure 2 Intra-operative view. (A) Under direct vision through the thoracotomy incision it is possible to measure the length from the skin incision to the apex of the pleural cavity and insert the chest tube correctly; (B) the thoracotomy wound can be closed in layers without interruptions in the deep planes; (C) the skin can be reconstructed with an intradermic suture allowing better post-operative aesthetic result. 

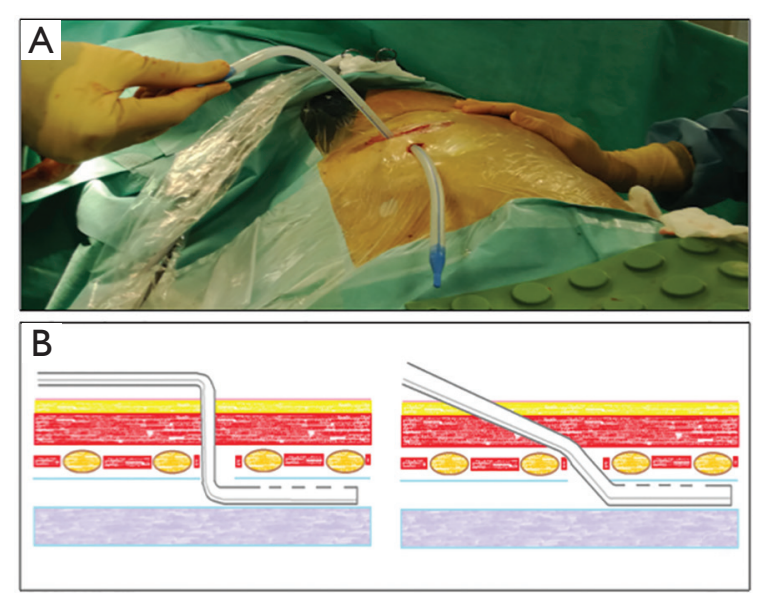

Figure 3 Comparison of chest tubes trajectories. (A) The picture shows the trajectories of the chest tube directly introduced through the thoracotomy access or using the dedicated tunnel that we propose. The difference in angle of entry results evident; (B) schematic reproduction of the insertion of the chest tube through the chest wall: on the left side the chest tube directly passed through the thoracotomy; on the right side the dedicated tunnel is used. The tunnel represents a stable fulcrum that avoids untoward angles preventing kinking and misplacements.

the drainage tube assumes with the classical technique in comparison to that that is gotten with ours; the different angle of entry of the drainage tube is rather evident.

In our experience, this simple shrewdness has resulted in a feasible and reproducible manoeuvre that allowed placing the chest tube easily and correctly with optimal functional results and complete lung re-expansion avoiding potential tube's kinking or misplacements. This observation is not applicable only in the case of major surgery but also, and perhaps more, in those minor interventions such as pleurodesis, where the correct contact between the pleurae in the early postoperative is fundamental.

We speculate that our technique can be at least comparable with the standard one in terms of postoperative pain or post-operative wound complications such as subcutaneous emphysema, infections or diastases. On the other hand, post-operative aesthetic results are comparable and even superior to the "single incision" approach, due to the possibility of a better reconstruction of the thoracotomy wound. Nevertheless, the aesthetic outcomes of a limited $1 \mathrm{~cm}$ skin access practiced for the tube insertion are negligible.

In conclusion, it should be never forgotten that minimal invasive surgery must remain a tool and not the goal of the surgical practice. With this assumption we support the minimal invasive uniportal surgery, but we would sacrifice the "single skin incision" assumption to a potentially increase safeness, stability and comfort for chest tube placement.

\section{Acknowledgements}

None.

\section{Footnote}

Conflicts of Interest: The authors have no conflicts of interest to declare.

\section{References}

1. Gonzalez-Rivas D. Uniportal thoracoscopic surgery: from medical thoracoscopy to non-intubated uniportal videoassisted major pulmonary resections. Ann Cardiothorac Surg 2016;5:85-91.

2. Harris CG, James RS, Tian DH, et al. Systematic review and meta-analysis of uniportal versus multiportal videoassisted thoracoscopic lobectomy for lung cancer. Ann Cardiothorac Surg 2016;5:76-84.

3. Sethuraman KN, Duong D, Mehta S, et al. Complications of tube thoracostomy placement in the emergency department. J Emerg Med 2011;40:14-20.

4. Gonzalez-Rivas D, Fieira E, Delgado M, et al. Uniportal video-assisted thoracoscopic lobectomy. J Thorac Dis 2013;5 Suppl 3:S234-45.

5. Satoh Y. Management of chest drainage tubes after lung surgery. Gen Thorac Cardiovasc Surg 2016;64:305-8.

Cite this article as: Palleschi A, Mendogni P, Mariolo AV, Nosotti M, Rosso L. An alternative chest tube placement after uniportal video-assisted thoracic surgery. $\mathrm{J}$ Thorac Dis 2018;10(5):3078-3080. doi: 10.21037/jtd.2018.04.108 